\title{
A chromosome-level reference genome of non- heading Chinese cabbage [Brassica campestris (syn. Brassica rapa) ssp. chinensis]
}

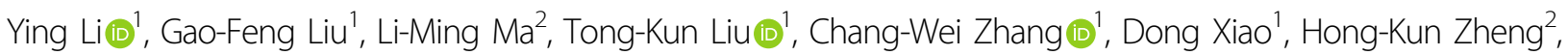
Fei Chen ${ }^{1}$ and Xi-Lin Hou (D)

\begin{abstract}
Non-heading Chinese cabbage (NHCC) is an important leafy vegetable cultivated worldwide. Here, we report the first high-quality, chromosome-level genome of NHCCO01 based on PacBio, Hi-C, and Illumina sequencing data. The assembled NHCC001 genome is $405.33 \mathrm{Mb}$ in size with a contig N50 of $2.83 \mathrm{Mb}$ and a scaffold N50 of $38.13 \mathrm{Mb}$. Approximately $53 \%$ of the assembled genome is composed of repetitive sequences, among which long terminal repeats (LTRs, 20.42\% of the genome) are the most abundant. Using Hi-C data, 97.9\% (396.83 Mb) of the sequences were assigned to 10 pseudochromosomes. Genome assessment showed that this B. rapa NHCC001 genome assembly is of better quality than other currently available B. rapa assemblies and that it contains 48,158 protein-coding genes, $99.56 \%$ of which are annotated in at least one functional database. Comparative genomic analysis confirmed that $B$. rapa NHCC001 underwent a whole-genome triplication (WGT) event shared with other Brassica species that occurred after the WGD events shared with Arabidopsis. Genes related to ascorbic acid metabolism showed little variation among the three B. rapa subspecies. The numbers of genes involved in glucosinolate biosynthesis and catabolism were higher in NHCCOO1 than in Chiifu and Z1, due primarily to tandem duplication. The newly assembled genome will provide an important resource for research on B. rapa, especially B. rapa ssp. chinensis.
\end{abstract}

\section{Introduction}

The Brassica genus comprises various economically important crops, many of which are extensively cultivated worldwide as oil crops and leafy vegetables. Six Brassica crop species comprise the "U's triangle", which includes the three basic diploid species $B$. rapa (A genome), $B$. nigra (B genome), and $B$. oleracea ( $\mathrm{C}$ genome), as well as the three amphidiploid species $B$. juncea (A and $\mathrm{B}$ genomes), B. napus (A and C genomes), and B. carinata (B

\footnotetext{
Correspondence: Xi-Lin Hou (hxl@njau.edu.cn)

${ }^{1}$ State Key Laboratory of Crop Genetics \& Germplasm Enhancement, Key Laboratory of Biology and Genetic Improvement of Horticultural Crops (East China), Ministry of Agriculture and Rural Affairs of the P. R. China, Engineering Research Center of Germplasm Enhancement and Utilization of Horticultural Crop, Ministry of Education of the P. R. China, College of Horticulture, Nanjing Agricultural University, Nanjing 210095, China

${ }^{2}$ Biomarker Technologies Corporation, Beijing 101300, China

These authors contributed equally: Ying Li, Gao-Feng Liu
}

and $C$ genomes) $)^{1}$. Brassica campestris, which is often used as a synonym for $B$. rapa, is an agronomically important species that includes various widely cultivated subspecies such as the turnip (ssp. rapa), Chinese cabbage (ssp. pekinensis), non-heading Chinese cabbage (ssp. chinensis), rapini (ssp. sylvestris), yellow sarson types (ssp. trilocularis), and oil types (ssp. oleifera $)^{2,3}$. Brassica campestris has been cultivated for specific phenotypic characteristics, such as enlarged edible roots, mid-ribs, leaves, and oil seeds. Non-heading Chinese cabbage (NHCC) is an important B. campestris (syn. B. rapa) subspecies that includes pak-choi (var. communis Tesn et Lee), Tacai (var. rosularis Tsen et Lee), Caitai (var. tsai-tai Hort.), Fenniecai (var. multiceps Hort.), and Taicai (var. tai-tsai Hort.). Pak-choi can be further divided into white petiole and green petiole types. It is one of the most popular vegetables in China, Vietnam, the Philippines, and other

\section{(c) The Author(s) 2021}

(c) (i) Open Access This article is licensed under a Creative Commons Attribution 4.0 International License, which permits use, sharing, adaptation, distribution and reproduction cc) in any medium or format, as long as you give appropriate credit to the original author(s) and the source, provide a link to the Creative Commons license, and indicate if changes were made. The images or other third party material in this article are included in the article's Creative Commons license, unless indicated otherwise in a credit line to the material. If material is not included in the article's Creative Commons license and your intended use is not permitted by statutory regulation or exceeds the permitted use, you will need to obtain permission directly from the copyright holder. To view a copy of this license, visit http://creativecommons.org/licenses/by/4.0/. 
East-Asian regions and is becoming increasingly popular around the world for its sweet, succulent, and nutritious leaves and stalks.

The genomes of two model plants, the dicot Arabidopsis and the monocot rice, were completed in 2000 and 2002 using early generation sequencing systems ${ }^{4,5}$. To date, approximately 200 plant genomes have now been published $^{6}$. In recent years, sequencing technologies have undergone tremendous development. Single-molecule sequencing, also referred to as third-generation sequencing, aims to meet the demand for high-quality plant genome assembly ${ }^{7}$, and PacBio and Oxford Nanopore Technology (ONT) sequencing have been used to assemble new, high-quality reference genomes for maize and tomato $^{8,9}$. The Brassica genus provides a good opportunity to study genome evolution in polyploids. The first $B$. rapa genome draft published in 2011 was assembled using a whole-genome shotgun strategy with Illumina short reads ${ }^{10}$. The recently released $B$. rapa Chiifu genome v3.0 based on PacBio sequencing lacked nearly $20 \%$ of the expected genome content ( 353.14 of $442.9 \mathrm{Mb})$, and the assembly was highly fragmented (contig N50 $1.45 \mathrm{Mb})^{11}$. The genome of a new morphotype, $B$. rapa $\mathrm{Z} 1$, was also assembled using Nanopore sequencing with a contig N50 of $5.51 \mathrm{Mb}^{12}$. Previously released $B$. rapa genomes provide great convenience for both genetic and comparative genomic studies, but they cannot fully satisfy the requirements of subsequent functional genomics research and the molecular breeding of non-heading Chinese cabbage. Therefore, it is necessary to assemble a high-quality reference genome for NHCC. In addition, current sequencing technologies are evolving rapidly, and the development of improved technologies enables the production of higher quality genomes.

Here, we present a chromosome-level assembly of $B$. rapa NHCC001 produced using a combination of PacBio sequencing and chromosome conformation capture (Hi$C)$ technologies. Our newly assembled $B$. rapa genome achieves a high level of continuity and completeness. It provides insights into the evolution of Brassica and constitutes an important resource for research, especially on the molecular mechanisms that underlie agricultural traits and on the breeding of B. rapa ssp. chinensis.

\section{Results}

De novo genome assembly

The size of the $B$. rapa NHCC001 genome estimated by $k$-mer analysis was $477.76 \mathrm{Mb}$, larger than that of the $B$. rapa Chiifu genome, which was estimated to be $442.90 \mathrm{Mb}$ (Supplementary Figure S1). The B. rapa NHCC001 genome heterozygosity rate was predicted to be about $0.17 \%$, its repeat sequence content about $58.57 \%$, and its GC content about $38.16 \%$. We performed the genome assembly of B. rapa NHCC001 using 5,387,116 high-quality PacBio sequencing reads $(61.44 \mathrm{~Gb})$ with an N50 of 16,938 bases. Details of the PacBio sequencing reads are provided in Supplementary Table S1. All reads were assembled by SMARTdenovo into an initial genome of $384.71 \mathrm{Mb}$, which represented $80.52 \%$ of the estimated B. rapa $\mathrm{NHCCO01}$ genome size. The assembled genome contained 891 contigs with a contig N50 of $2.13 \mathrm{Mb}$ and a maximum contig length of $16.11 \mathrm{Mb}$.

$\mathrm{Hi}-\mathrm{C}$ data were used to assign the resulting contigs to their chromosomal positions. We generated $68.69 \mathrm{~Gb}$ of clean data with $\sim 143 \times$ coverage and anchored the assembled contigs to ten pseudochromosomes using the $\mathrm{Hi}-\mathrm{C}$ data. The final chromosome-scale genome was composed of ten clusters, as indicated in the $\mathrm{Hi}-\mathrm{C}$ interaction heat map (Supplementary Fig. S2). The anchored $97.90 \%(396.83 \mathrm{Mb})$ of assembled genome content included 553 contigs clustered by Hi-C data (Fig. 1, Supplementary Table S2). Among these clustered contigs, 300 $(363.76 \mathrm{Mb})$ were anchored with defined order and orientation (Supplementary Table S2). The gap-closing step for pseudochromosomes was performed using errorcorrected Nanopore clean reads (Supplementary Table $\mathrm{S} 1)$. The final chromosome-scale genome was $405.33 \mathrm{Mb}$ in length with 602 contigs (contig $\mathrm{N} 50=2.83 \mathrm{Mb}$ ) and contained 312 scaffolds with a scaffold N50 of $38.13 \mathrm{Mb}$ and 290 gaps (Table 1). The final genome assembly represented $84.83 \%$ of the estimated B. rapa NHCC001 genome, compared with $79.73 \%$ and $74.20 \%$ for the recently released Chiifu v3.0 and Z1 genomes, respectively. The GC content of the assembled genome was $37.13 \%$, similar to the $38.16 \%$ estimate from $k$-mer analysis (Table 1).

\section{Completeness and accuracy of the assembly}

BUSCO v4.0.6 analysis revealed that $99.07 \%$ (1559 of 1614) of the core eukaryotic genes-including 1361 single-copy orthologs and 238 duplicated orthologswere present in our assembly (Supplementary Table S3). The base error percentage of the genome assembly was estimated to be $0.0011 \%$ (Supplementary Table S4). More than $99.96 \%$ of the full-length transcripts assembled de novo from transcriptome data had best hits on single contigs, confirming the completeness of the genome (Supplementary Table S5). In addition, $89.05-91.19 \%$ of the clean reads from four RNA-seq libraries could be uniquely mapped to the genome (Supplementary Table S6). Taken together, these independent assessments confirmed that the B. rapa NHCCOO1 genome had high contiguity, completeness, and base accuracy.

\section{Genome annotation}

A combination of de novo, homology-based, and transcriptome-based predictions indicated that $102.05 \mathrm{Mb}$ (25.18\%) of the $405.33 \mathrm{Mb}$ assembled genome encoded 


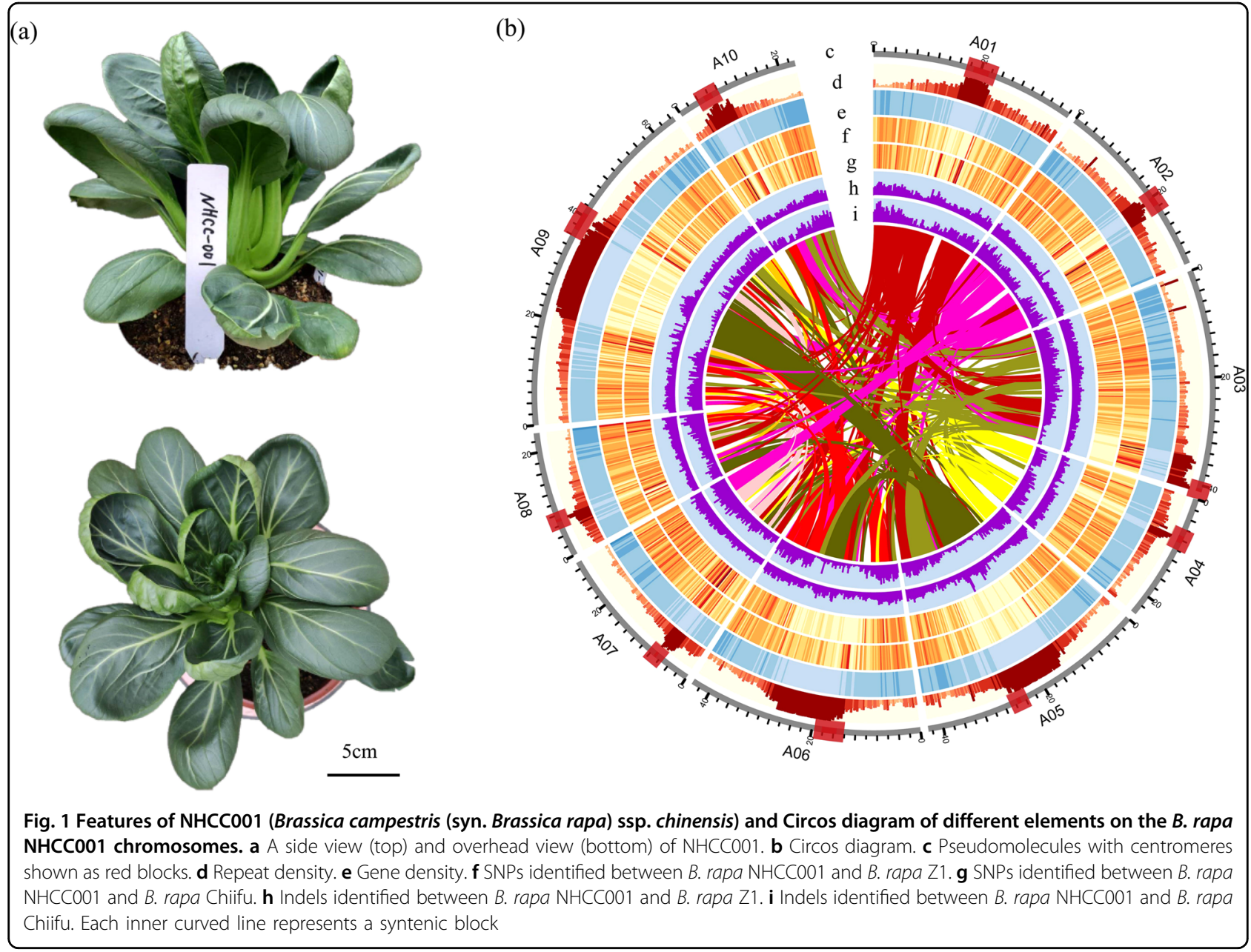

48,158 genes with a mean exon number of 5.08 per gene, similar to $A$. thaliana and $B$. rapa. The average gene length was $2118.99 \mathrm{bp}$, and the average exon and intron lengths were 1236.80 and 873.19 bp per gene, respectively. Among the 48,158 predicted genes, 47,872 (99.41\%) could be annotated using at least one functional protein database (Supplementary Table S7). The vast majority of genes (97.88\%; 47,135 of 48,158) were anchored on chromosomes, and only $2.12 \%$ (1023 of 48,158) were located on scaffolds. We used the newly assembled NHCC001 genome as a reference to measure the expression levels of 48,159 annotated genes (Supplementary Table S18) and found that 25,056 and 21,662 genes were expressed in leaf and root tissues, respectively, with FPKM $\geq 1$.

Homology-based and de novo approaches were also used to search for and predict repetitive sequences. A total of $213.04 \mathrm{Mb}(52.56 \%)$ of the assembled NHCC001 genome comprised repetitive sequences, a percentage higher than that of the previous B. rapa Chiifu v3.0 assembly (37.93\%, 133.95 Mb) (Supplementary Table S8). Among these repetitive elements, LTR retrotransposons were the most abundant, accounting for $20.42 \%$ of the genome, followed by DNA transposons (5.37\%), LINEs (3.20\%), and SINEs (0.29\%) (Supplementary Table S8). In addition, $1.19 \%(4.86 \mathrm{Mb})$ of the assembled genome was annotated as non-coding RNA, including 151 miRNAs, 1,361 tRNAs, 3,907 rRNAs, and 1,272 snoRNAs.

\section{Evolution of the NHCC001 genome}

The genome sequences of 11 plant species and two other B. rapa subspecies were collected and used for comparative genomic analysis with $\mathrm{NHCC001}$ to investigate its genome evolution and divergence. We clustered the annotated NHCC001 genes with those from the other plant genome using OrthoMCL. Of the 48,158 proteincoding genes in the NHCC001 genome, 46,316 were grouped into 27,536 gene families with an average of 1.68 genes per family. There were 7835 common gene families and 165 NHCC001-specific families (Fig. 2c, Supplementary Table S9). Furthermore, we found that 1771 gene families had expanded and 2051 had contracted in the newly assembled NHCC001 genome (Fig. 2b). The 165 NHCC001-specific gene families contained 423 genes, 
Table 1 Statistics for the de novo assembled NHCC001 genome and other current $B$. rapa genome versions

\begin{tabular}{llll}
\hline & $\begin{array}{l}\text { B. rapa (ssp. } \\
\text { chinensis) }\end{array}$ & $\begin{array}{l}\text { B. rapa (ssp. } \\
\text { pekinensis) }\end{array}$ & $\begin{array}{l}\text { B. rapa (ssp. } \\
\text { trilocularis) }\end{array}$ \\
\hline Accession & NHCC001 & Chiifu (v3.0) & $Z 1$ \\
$\begin{array}{l}\text { Estimated genome } \\
\text { size (Mb) }\end{array}$ & 477.76 & 442.90 & 529.00 \\
$\begin{array}{l}\text { Genome sequence } \\
\text { size (Mb) }\end{array}$ & 405.33 & 353.14 & 392.50 \\
Contig number & 602 & 1498 & \\
Contig N50 (Mb) & 2.83 & 1.45 & 627 \\
Contig max (Mb) & 22.49 & 9.4 & 22.12 \\
Scaffold number & 312 & 1301 & 335 \\
Scaffold N50 (Mb) & 38.13 & 4.44 & 15.38 \\
GC content (\%) & 37.13 & 36.83 & 37.20 \\
Gaps total & 13,382 & 2,078 & 32,966 \\
length (kb) & & & 46,721 \\
Annotated & 48,158 & 46,250 & \\
gene number & & & \\
\hline
\end{tabular}

and 1501 of the 7835 common gene families contained one copy in each plant. We used these 1501 single-copy orthologs for phylogenetic analysis based on the maximum likelihood method. B. rapa $\mathrm{NHCCO01}$ and B. rapa Chiifu, which derive from the common ancestral genome of Brassica species, were clustered together on a branch (Fig. 2a). Using B. oleracea as an outgroup, evolutionary rates and protein mutation sites were compared among the three $B$. rapa genomes to add confidence to the genomic analysis (Supplementary Fig. S4). The three subgenomes of $B$. rapa diverged approximately 1.0-2.3 Mya, and the data further confirmed that the Brassica ancestor diverged from Thellungiella parvula approximately 20.9-23.4 Mya (Fig. 2a).

4DTv and Ks values of gene pairs were estimated based on the syntenic blocks detected among NHCC001 and other plant genomes (Fig. 3a, Supplementary Fig. S3). The results indicated that a recent WGT event at $4 \mathrm{Dtv} \approx 0.15$ (Ks peak value $\approx 0.3$ ), which was previously reported as a Brassicaceae-specific triplication $(\mathrm{Br}-\alpha-\mathrm{WGD})^{13-15}$, had also occurred in the evolutionary history of NHCC001 (Fig. 3a). Furthermore, we confirmed the almost complete triplication of the B. rapa NHCC001 genome relative to those of Thellungiella halophila and A. thaliana

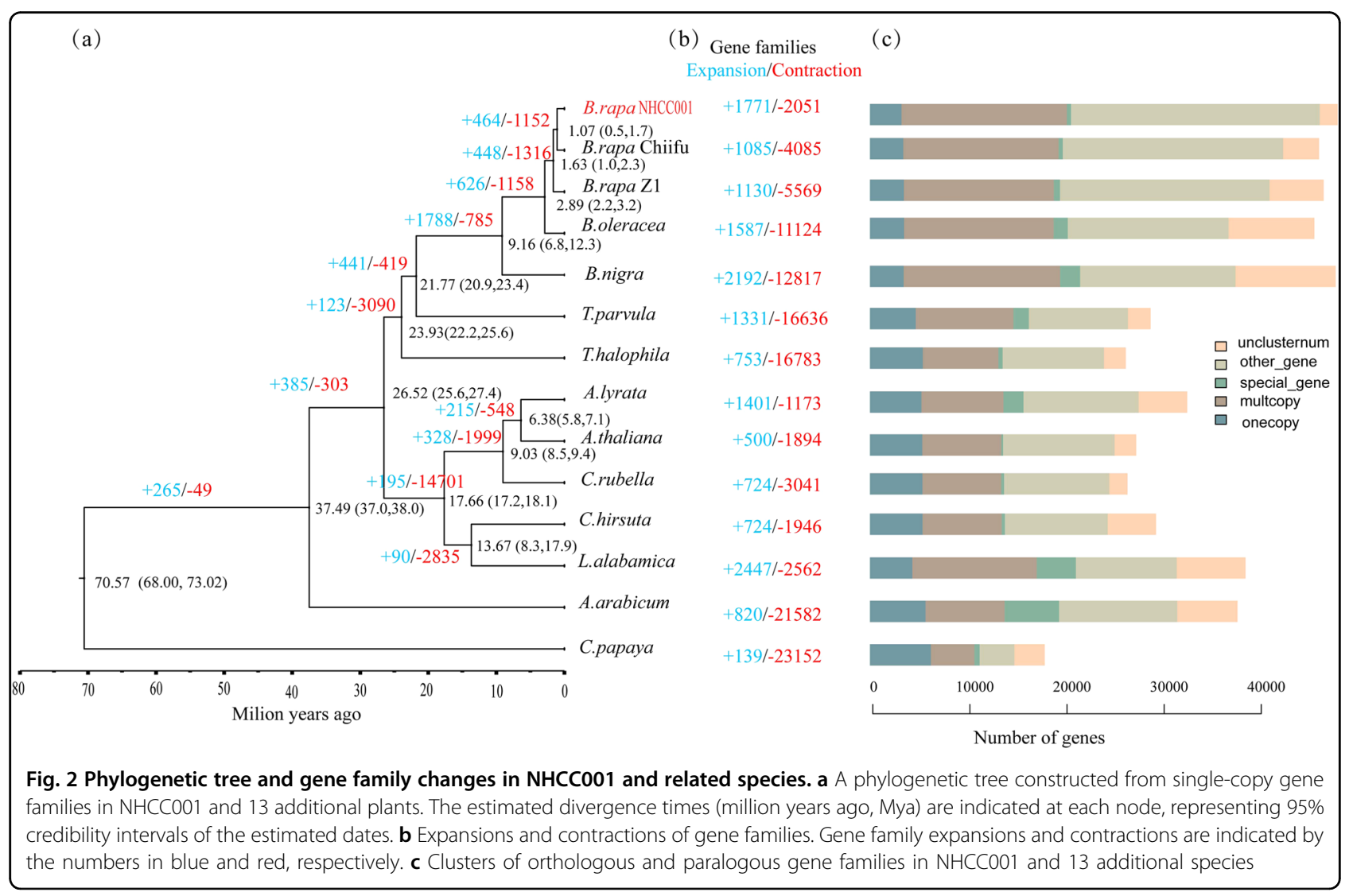



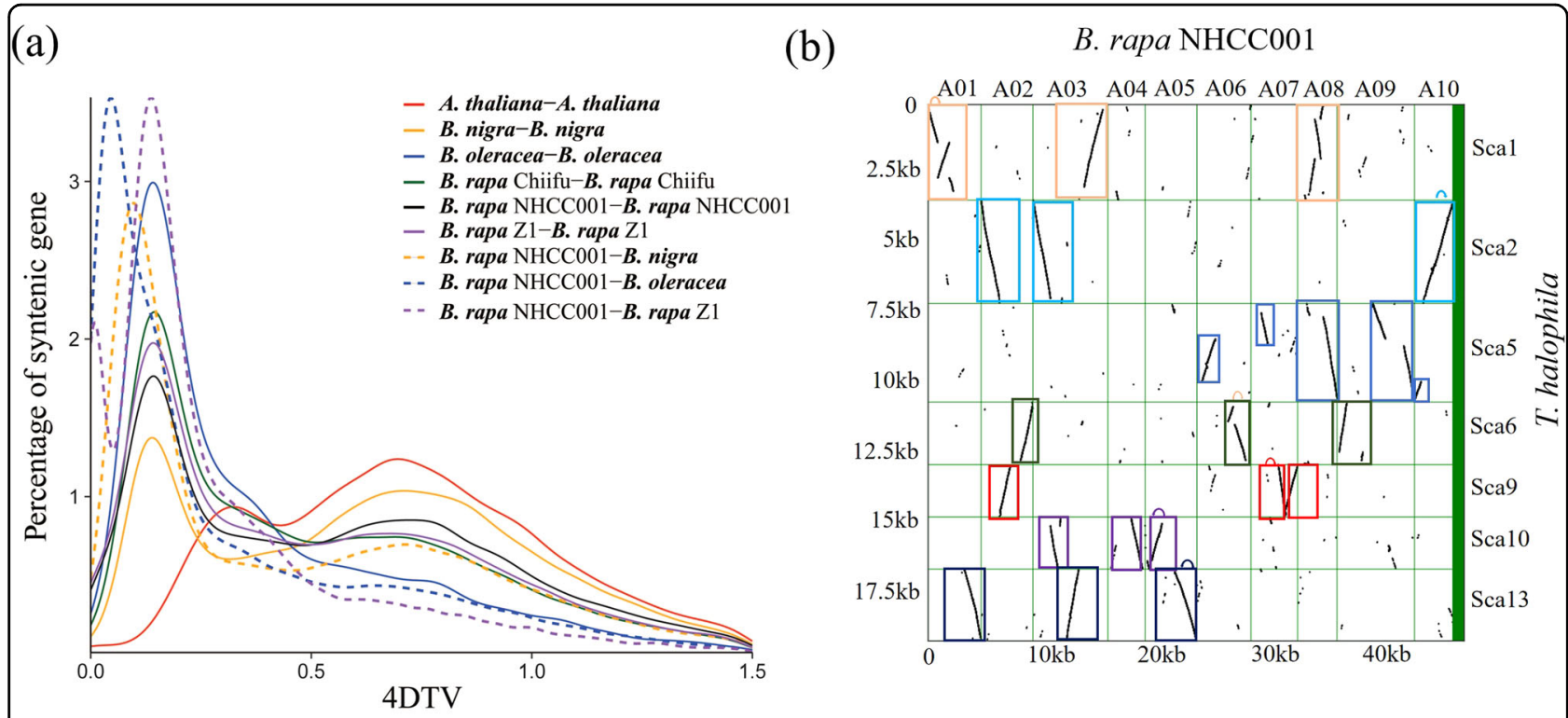

(c)

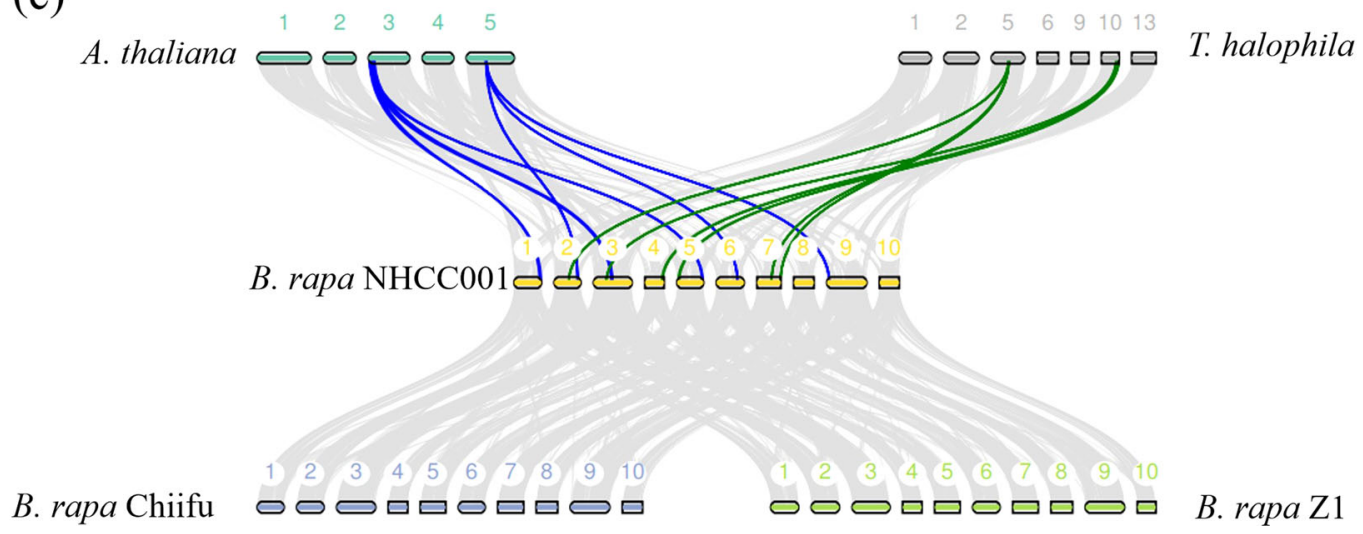

Fig. 3 Evolution of the NHCC001 genome. a Four-fold synonymous third-codon transversion rate (4Dtv). 4dTv distance distribution of duplicated gene pairs in syntenic blocks within the genomes of B. rapa NHCC001, B. rapa Chiifu, B. rapa Z1, B. oleracea, B. nigra, and A. thaliana. b Segmental collinearity of the B. rapa NHCCO01 and T. halophila genomes. Conserved collinear blocks of gene models between the ten chromosomes of $B$. rapa NHCCOO1 (horizontal axis) and the eight longest scaffolds of T. halophila (vertical axis) are shown. c Syntenic blocks between B. rapa NHCC001 and four other Brassicaceae species. The numbers indicate the corresponding chromosomes or scaffolds in each species

(Fig. 3b-c). The divergence of B. rapa NHCCOO1 and $B$. nigra occurred at a peak of $\sim 0.10$, followed by that of $B$. rapa $\mathrm{NHCC001}$ and $B$. oleracea $(4 \mathrm{dTv}=0.05)$, consistent with the phylogenetic analysis (Fig. 3). The genomes of $B$. rapa $\mathrm{NHCC001,} \mathrm{B.} \mathrm{rapa} \mathrm{Z1}$, and $B$. rapa Chiifu arose recently and did not exhibit significant divergence compared with the A and B genomes of Brassica species (Supplementary Fig. S3). These findings were consistent with the phylogenetic analysis of Brassica and other representative species.

\section{Chromosome structure of the $B$. rapa NHCC001 genome}

Previous studies have proposed a model for comparative genomics and chromosomal analyses based on the concept of the Ancestral Crucifer Karyotype (ACK; $n=8$ ), which comprises eight chromosomes and 24 genomic blocks (GBs, named A to $\mathrm{X})^{16}$. Syntenic orthologs between NHCC001 and A. thaliana were identified first ${ }^{17}$, and the three subgenomes were identified based on the syntenic relationship between $\mathrm{NHCCO01}$ and A. thaliana (Supplementary Table S19). Subgenomes were ordered based on gene densities from high to low and were named LF, MF1, and MF $2^{17}$. As expected, all 72 genome blocks $(3 \times 24)$ in the NHCC001 genome were identified, compared with 71 identified in Chiifu v3.0 and previous versions $^{18}$ (Fig. 4). Compared with the distribution of genome blocks in Chiifu V3.0, most were arranged similarly in NHCC001. The lost genome block G (MF2) in 


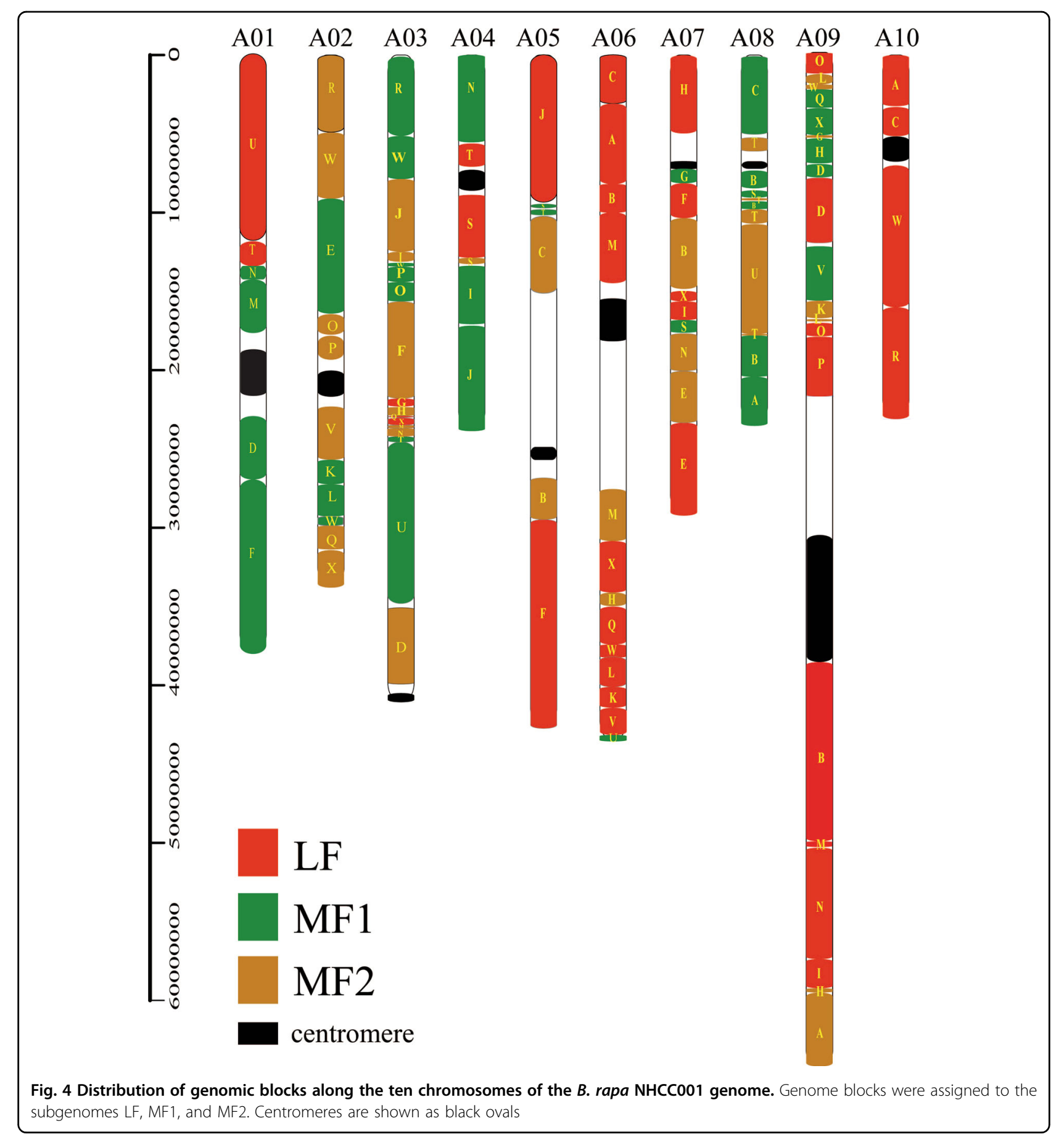

Chiifu was identified on chromosome A09 between X (MF1) and H(MF1) in NHCC001 (Fig. 4, Supplementary Table S20). In addition, we identified two new fragmented genome blocks: $\mathrm{T}(\mathrm{MF} 1)$ and $\mathrm{O}(\mathrm{LF})$ were identified on chromosomes A05 and A09, respectively, but were not observed in Chiifu V3.0. Two new fragmented genome blocks, F (LF) and F (MF1) on chromosomes A01 and A05 in Chiifu V3.0, were not identified in NHCC001 or in ref. ${ }^{10,18}$. We counted the gene numbers in the three subgenomes and found 13,283, 9011, and 7419 genes in the LF, MF1, and MF2 subgenomes, respectively, that were syntenic to $A$. thaliana.

\section{Global genome comparisons of three $B$. rapa genomes}

SNPs and indels were identified between $B$. rapa NHCC001, B. rapa Z1, and B. rapa Chiifu (Fig. 1, 
Supplementary Table S10). A total of $1,718,037$ SNPs and 738,275 indels were identified between $B$. rapa NHCC001 and $B$. rapa $\mathrm{Z1}$, and $1,305,874$ SNPs and 469,629 indels were identified between $B$. rapa $\mathrm{NHCC001}$ and $B$ rapa Chiifu. We then compared the NHCC001 genome sequence to those of Chiifu and $\mathrm{Z} 1$ and identified a large number of syntenic regions (Fig. 3c). A total of 534 syntenic blocks were detected between $B$. rapa $\mathrm{NHCC001}$ and $B$. rapa Chiifu, comprising 49,166 gene pairs. Likewise, 557 syntenic blocks were detected between $B$. rapa NHCC001 and B. rapa Z1, comprising 48,976 gene pairs (Fig. 3c). Tandem gene arrays were also identified in the three genomes using SynOrths ${ }^{19}$. A total of 2211 tandem arrays (corresponding to 5296 tandemly duplicated genes) were identified in $B$. rapa NHCC001. By contrast, more tandem arrays (2317 arrays, 5584 genes) were identified in the $B$. rapa Chiifu genome and fewer (2013 arrays, 4781 genes) in the $\mathrm{Z} 1$ genome.

We identified 10,851 NHCC001-specific genomic segments $(\sim 13 \mathrm{Mb})$ and 8496 Chiifu-specific genomic segments $(\sim 10 \mathrm{Mb})$ longer than $500 \mathrm{bp}$ (Supplementary Table S11). Most (98.7\%) of these PAV (presence and absence variation) sequences were shorter than $5 \mathrm{~kb}$, although 222 and 177 PAV sequences were longer than $5 \mathrm{~kb}$ in NHCC001 and Chiifu, respectively (Supplementary Table S11). Three NHCC001-specific sequence clusters (compared with Chiifu) on chromosomes 7 and 2 contained 132 predicted genes, and eight NHCC001specific sequence clusters (compared with Z1) on eight separate chromosomes contained 596 predicted genes (Supplementary Table S12). Details of PAV sequences and clusters identified between B. rapa $\mathrm{NHCC001}$ and B. rapa $\mathrm{Z} 1$ are shown in Supplementary Table S13. Among the PAV genes identified between NHCC001 and Chiifu, 125 were specific to NHCC001, and 369 were specific to Chiifu (Supplementary Table S21). These specific segments and genes may contribute to the diversity of the three $B$. rapa subspecies.

\section{Leaf adaxial-abaxial patterning genes in $B$. rapa}

The most significant difference between NHCC001, a non-heading Chinese cabbage cultivar, and Chiifu, the heading Chinese cabbage, is the heading trait. Leaf incurvature, controlled by multiple genes, is an essential prerequisite for the formation of a leafy head ${ }^{20,21}$. Previous studies have identified adaxial-abaxial (ad-ab) patterning genes and investigated their genetic variation to uncover the mechanisms that underlie leaf incurvature during head formation in heading $B$. rapa and $A$. thali$a n a^{22}$. Using 26 homologs from $A$. thaliana, we identified 51, 47, and 49 leaf ad-ab patterning genes in NHCC001, Chiifu v3.0, and Z1, respectively (Supplementary Table S14). Copy number variation was found among the three B. rapa genomes. In non-heading $\mathrm{NHCCO01}$ and $\mathrm{Z} 1$, three homologs of AtARF4 were identified (Supplementary Table S14), compared with only two AtARF4 homologs in heading Chinese cabbage Chiifu. Previous studies have shown that arf3 arf4 double mutants develop leaves that are curled up and resemble the phenotype of kan 1 kan2 leaves, indicating an overlap in the function of leaf abaxial polarity ${ }^{23-25}$. Furthermore, there were two homologs of AtAGO7 in NHCC001 and Z1 but only one AtAGO7 homolog in Chiifu. Two AtDCL1 homologs in Chiifu were identified as tandem duplicates. Copy number variation in ab-ad genes may therefore contribute to leaf head formation in Chinese cabbage.

\section{Identification of genes involved in ascorbic acid and glucosinolate metabolic pathways}

Pak-choi is well known for its high nutritional value, particularly its abundant contents of ascorbic acid (AsA, vitamin C) and glucosinolates (GSLs). A previous study reported that the leaf AsA concentration in NHCC001 was $\sim 110 \mathrm{mg} / 100 \mathrm{~g} \mathrm{FW}^{26}$. Using the newly assembled NHCC001 genome and the sequences of AsA-related genes from $A$. thaliana, we identified and compared genes involved in AsA biosynthesis and recycling from $B$. rapa NHCC001, B. rapa Chiifu, and B. rapa Z1 (Fig. 5, Supplementary Table S15). A total of 87, 93, and 93 AsArelated genes were identified in the B. rapa NHCC001, Chiifu, and Z1 genomes, respectively (Supplementary Table S15). Four regions of homeologs had undergone tandem duplication. The numbers of AsA-related homologs were highly consistent among the three $B$. rapa genomes; most were located on conserved collinear blocks and showed little variation among the three subspecies. The expression patterns of these genes were measured in the root and leaf tissues of NHCCO01 (Fig. 5). Two GGalPP homologs and one IPS homolog were highly expressed in the leaf (Fig. 5), whereas some $A P X$, $M D A R$, and $D H A R$ homologs from the recycling pathway were highly expressed in both roots and leaves (Fig. 5).

Glucosinolates and their hydrolysis products have an important role in human health and plant defence ${ }^{27}$. More than 130 structurally distinct GSLs are present in 16 families of dicotyledonous angiosperms ${ }^{27,28}$, and more than 14 different GSLs have been identified and quantified in the young leaves of different $B$. rapa varieties ${ }^{29}$. Using $A$. thaliana GSL genes as queries, GSL genes were identified in B. rapa NHCC001, Chiifu, and Z1 (Supplementary Table S16). Interestingly, GSL gene numbers were highly expanded in the $B$. rapa genome, with a high proportion of tandem genes. GSL biosynthesis and catabolism are probably similar among the three sequenced $B$. rapa species, but there was nonetheless substantial variation in the proportion of tandem genes such as MAM1, ST5b, FMOGS-OX, AOP, TGGs, and NSPs (Supplementary Table S16). Flavin-monooxygenase FMOGS-OX 


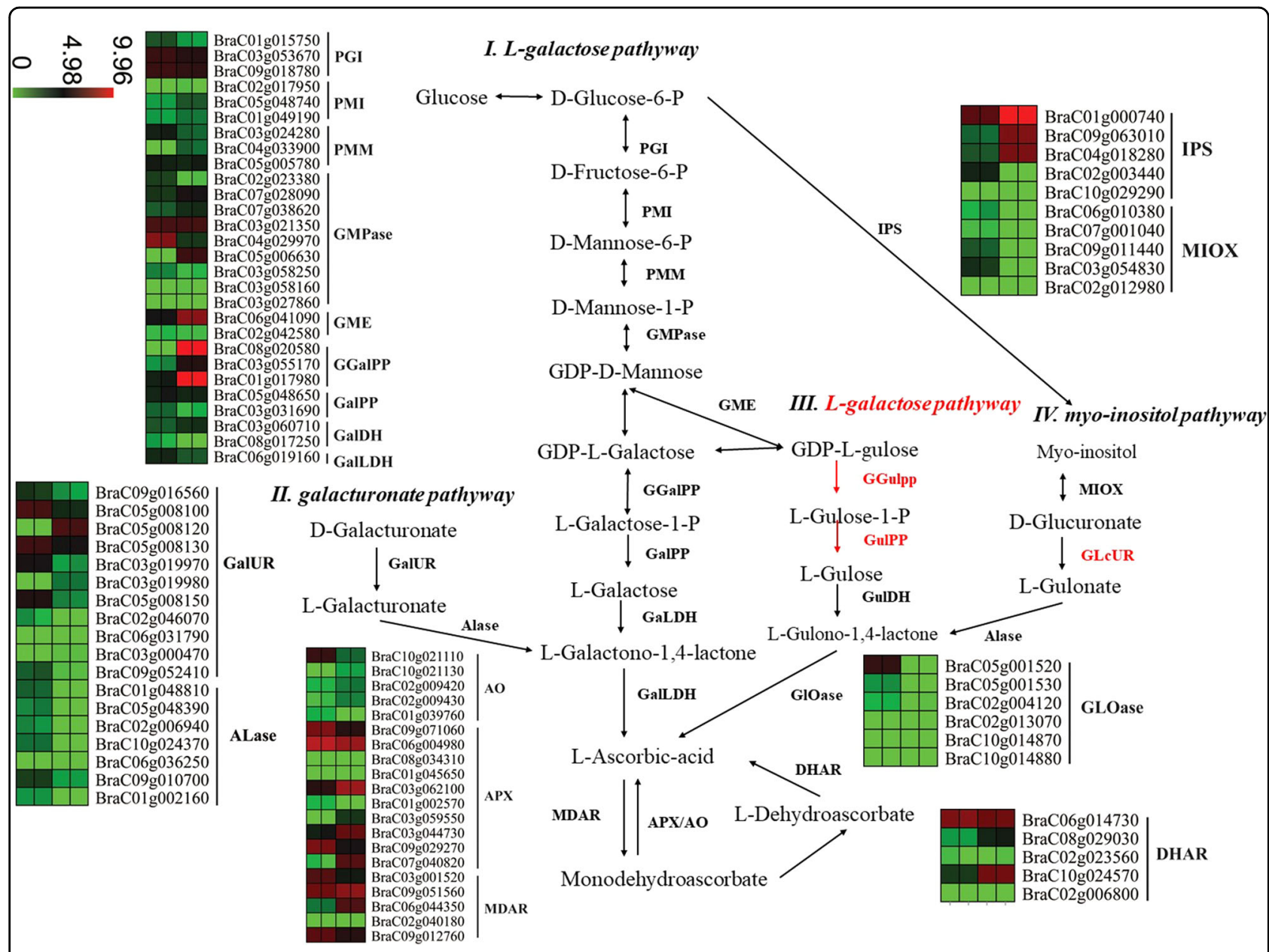

Fig. 5 Genes involved in the biosynthesis and recycling of ascorbic acid (AsA, vitamin C) and their expression patterns in B. rapa NHCC001. Red lines indicate hypothetical reactions. Heatmaps show log2-transformed expression values of ASA-related genes in Root-1, Root-2, Leaf-1, and Leaf-2 (from left to right)

catalyzes the S-oxygenation of methylthioalkyl to methylsulfinylalkyl GSLs during the biosynthesis of aliphatic GSLs in Arabidopsis thaliana ${ }^{30}$. We identified six, three, and three FMOGS-OX homologs in NHCC001, Chiifu, and Z1, respectively (Supplementary Table S16). Phylogenetic analysis showed that the FMOGS-OX genes were clustered into four clades, one of which included only three FMOGS-OX genes from B. rapa NHCC001 (Fig. 6a). Gene expression analysis showed that BraC09g068950 in clade I and BraC09g014640 in clade II were highly expressed in both roots and leaves, whereas BraC09g014670 had the highest leaf expression of all the FMOGS-OX genes. TGG catalyzes the hydrolysis of GSLs into compounds that are toxic to various microbes and herbivores ${ }^{31}$. TGG homologs were identified in the three $B$. rapa genomes and used to construct a phylogenetic tree (Fig. 6b, Supplementary Table S16). To our surprise, only one TGG homologue was initially identified in the $B$. rapa Chiifu v3.0 genome, despite the fact that nine TGG homologs were identified in the previous $B$. rapa Chiifu genome version ${ }^{5}$. We speculated that this may have been caused by a gene prediction error in Chiifu v3.0. We therefore searched the Chiifu v3.0 genome using four $A$. thaliana TGGs as queries and found 17 new TGG genes. Detailed information on these genes is provided in Supplementary Table S17. There were 21 TGGs in B. rapa NHCC001. Two tandem duplicates, $\mathrm{BraCO} g 043340$ and BraC02g043360, both showed high expression in NHCC001 leaves (Fig. 6b).

\section{Discussion}

Brassica rapa species can be grouped into six subspecies: turnips, sarsons, turnip rapes, and the Japanese group, which includes pak-choi and heading Chinese cabbages $^{32}$. Although they originated from the same ancestor, these varieties show very high morphotype diversity. To date, only one cultivar of heading Chinese cabbage $(\text { Chiifu-401-42) })^{10}$ and one sarson type $(\mathrm{Z} 1)^{12}$ 
(a)

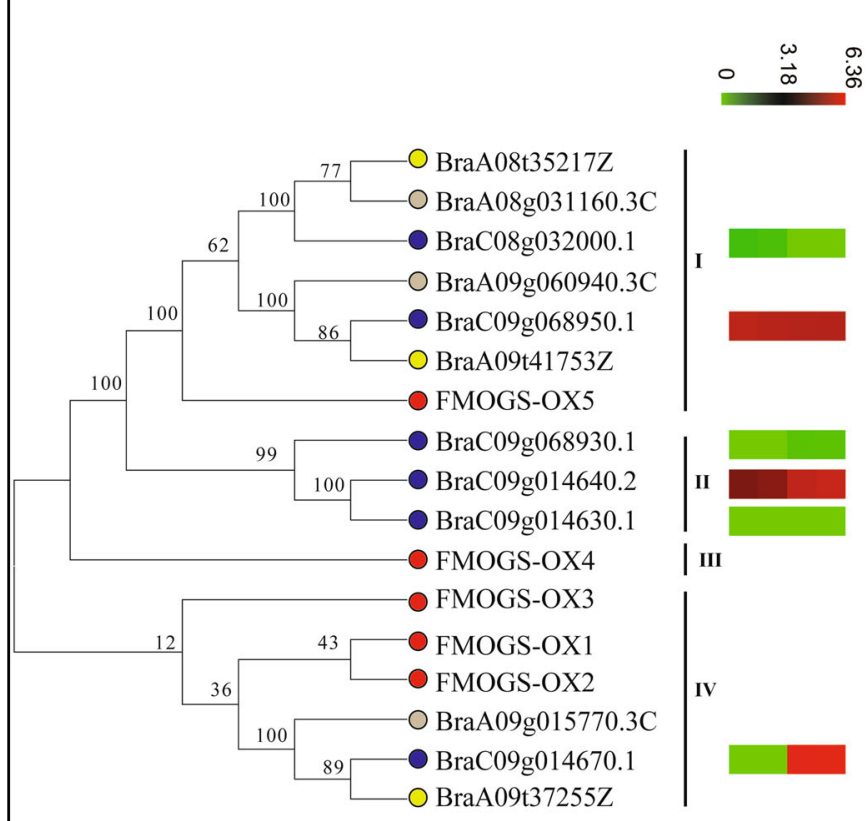

(b)

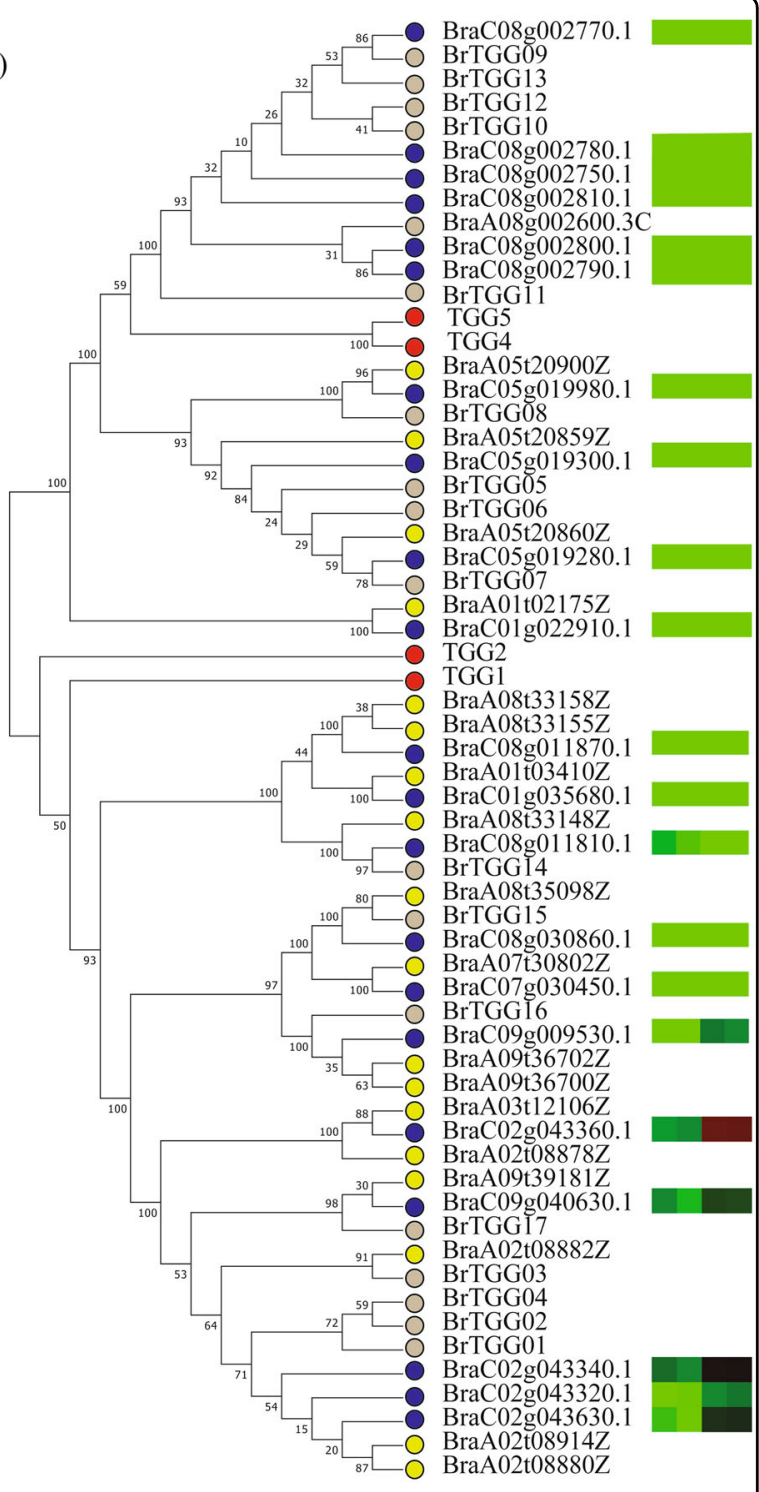

Fig. 6 Phylogenetic relationships and expression patterns of the FMOGS-OX and TGG genes. Phylogenetic tree of the FMOGS-OX (a) and TGG (b) genes in B. rapa NHCCOO1 and three related species, and their expression patterns in B. rapa NHCC001. The NJ tree was constructed with MEGA using the full-length amino acid sequences. The solid red, blue, gray, and yellow dots represent $A$. thaliana, B. rapa NHCC001, B. rapa Chiifu, and B. rapa Z1, respectively. Heatmaps show log2-transformed expression values of FMOGS-OX and TGG genes in Root-1, Root-2, Leaf-1, and Leaf-2 (from left to right)

have been sequenced. The scarcity of genomic resources has hindered research on the evolution of non-model plant species and the genetic basis of phenotypic diversity. In the present study, we assembled a high-quality genome of the pak-choi cultivar 'Suzhouqing' (NHCC001) using a combination of PacBio and $\mathrm{Hi}-\mathrm{C}$ data. The assembly covers approximately $84.84 \%$ of the estimated NHCC001 genome. Use of PacBio and $\mathrm{Hi}-\mathrm{C}$ technologies provided a high-quality assembly in terms of contiguity and completeness of genic and repetitive regions. More repeat sequences $(213.04 \mathrm{Mb})$ were identified in the present study than in previously published $B$. rapa genomes (Table 1). A total of 48,158 genes were identified, more than those identified in the B. rapa Chiifu $(45,985)^{11}$ and B. rapa $\mathrm{Z} 1(46,721)^{12}$ assemblies. Our assembly represents a real improvement of the $B$. rapa genome, particularly for regions enriched in repetitive elements, and provides a valuable resource for comparative genomics and evolutionary studies.

Many efforts have been made to resolve the relationships among subspecies and the domestication history of B. rapa. Chen et al. and Qi et al. also suggested that the 
Chinese cabbage group was positioned at the most distant point from the $B$. rapa root and was most divergent from the last $B$. rapa common ancestor ${ }^{2,32}$. Our phylogenetic analysis further confirmed that the sarson type diverged from the last $B$. rapa common ancestor earlier than Chinese cabbage and pak-choi (Fig. 2). Comparative genomic analysis revealed variations in the three $B$ rapa genomes; numerous intraspecific variations such as SNPs, indels, and PAVs were revealed. Copy number variation was also identified in adaxial-abaxial patterning genes, which may lead to a better understanding of the molecular mechanisms that underlie the leaf heading trait in $B$. rapa. Based on homolog searches, we identified candidate AsA-related and GSL-related genes in the three B. rapa genomes. This more complete genome assembly will provide a solid basis for future metabolism-related studies.

\section{Conclusion}

Our newly assembled $B$. rapa genome achieves a high level of continuity and genic completeness. The new $B$. rapa genome will be of great help in understanding the evolution of the Brassicas and will provide an important resource for research, especially the molecular investigation of agricultural traits and the breeding of B. rapa ssp. chinensis.

\section{Materials and methods}

\section{Illumina sequencing of short paired-end (PE) reads}

The Brassica campestris (syn. Brassica rapa) ssp. chinensis ('Suzhouqing', green petiole type pak-choi) selfinbred line NHCC001 was used for genome sequencing. Genomic DNA was extracted from NHCC001 leaf tissue and fragmented. One library was constructed and sequenced on the Illumina platform (Illumina, San Diego, CA, USA), and $76.35 \mathrm{~Gb}$ of clean reads were generated after trimming adaptors and low-quality bases and removing mitochondria and chloroplast DNA contamination. These data were used for genome estimation, gap closing, assembly polishing, and completeness assessment of the final assembly. Genome size was estimated using the formula: Genome Size=total $k$-mer number/average peak depth using $k$-mer analysis.

\section{RNA sequencing}

Root, stem, leaf, and flower tissues were harvested and immediately frozen in liquid nitrogen. Total RNA was extracted using the TRIzol reagent (Invitrogen, USA) following the manufacturer's instructions and pooled for sequencing. SMRTbell libraries were constructed using the PacBio DNA Template Prep Kit 2.0 and then sequenced on the PacBio RS II platform. In addition, cDNA libraries were constructed from roots and leaves and used for second-generation sequencing on the
Illumina HiSeq 2500 platform to generate 125-bp pairedend reads following the manufacturer's protocol.

\section{Pacific Biosciences (PacBio) sequencing and de novo assembly of PacBio subreads}

Genomic DNA was isolated and extracted from fresh NHCC001 leaves using the CTAB method ${ }^{33}$. Quality control of genomic DNA was performed using a Nanodrop spectrophotometer. 10 ug of gDNA was sheared to $\sim 20-\mathrm{kb}$ targeted size using a Covaris g-TUBE, and a 20-kb insert library was constructed following the standard PacBio protocol. SMRT cells were sequenced on the PacBio RSII platform (PacBio Sequel sequencer at Biomarker Technologies Corporation) with P6-C4 chemistry. PacBio subreads were corrected, trimmed, and assembled using SMARTdenovo (5cc1356) (https://github.com/ ruanjue/smartdenovo.git). Sequencing errors in subreads were corrected using Canu (v1.5) ${ }^{34}$ with the settings genomeSize $=480,000,000$, corOutCoverage $=100$, and correctedErrorRate $=0.025$. Finally, to ensure basepairing accuracy of the assembly results, we further polished the consensus sequence based on errorcorrected PacBio subreads using Arrow (v2.3.3) with the parameter $-\mathrm{j} 4$ and Illumina PE reads using Pilon (v1.22) ${ }^{35}$ with the parameters --mindepth 10, --changes, --threads 4 , and --fix bases.

\section{Hi-C library sequencing}

$\mathrm{Hi}-\mathrm{C}$ libraries were prepared from NHCC001 leaves as described previously ${ }^{36}$ and sequenced on the Illumina HiSeq X Ten platform $(2 \times 150 \mathrm{bp})$ to generate $229,427,069$ paired-end reads. The Hi-C data were mapped to PacBiobased contigs using BWA (v0.7.10-r789; aln mapping method) with the parameters -M 3, -O 11, -E 4, and -t 8 . HiC-Pro (v2.8.1) ${ }^{37}$ was used for duplicate removal and quality control. Using contigs assembled from PacBio data, $\mathrm{Hi}-\mathrm{C}$ data were used to correct mis-joins in contigs and to order and orient contigs. Pre-assembly was performed for contig correction by splitting contigs into segments and then pre-assembling the segments with $\mathrm{Hi}$ $\mathrm{C}$ data. Misassembled points were defined and broken when split segments could not be placed into the original position. Finally, the corrected contigs were assembled using LACHESIS with parameters CLUSTER_MIN_RE_SITES $=30, \quad$ CLUSTER_MAX_LINK_DENSITY $=2$, ORDER_MIN_N_RES_IN_TRUN $=25$, and ORDER_MIN_N_RES_IN_SHREDS $=26$ with $\mathrm{Hi}-\mathrm{C}$ valid pairs. Gaps between ordered contigs were filled with 100 Ns. To improve the contiguity of the assembly results, a gapclosing step for pseudochromosomes was performed using PBJelly $(\mathrm{v} 15.2 .20)^{38}$ with the parameter --min$\mathrm{Gap}=1$ using error-corrected Nanopore clean reads. The contact map was visualized with heatmaps at a $100-\mathrm{kb}$ resolution. 


\section{Genome assembly evaluation}

The completeness of the assembly was evaluated based on the full-length transcriptome and PE reads using Tophat $2^{39}$ with default parameters and BWA (v0.7.10) ${ }^{40}$ with parameters -t 4 and $-\mathrm{M}$, respectively. The corrected PacBio subreads were also used for genome evaluation with blasr (v1.3.1) (https://github.com/PacificBiosciences/blasr) using parameters -bestn 1, -minPctIdentity 70, and -nproc 4 . BUSCO v4.0.6 and CEGMA (v2.5) were also used to assess assembly completeness. BUSCO ${ }^{41}$ was run using the embryophyta_odb10 dataset with default parameters.

\section{Annotation of transposable elements (TEs)}

Transposable elements were identified using de novo and homology-based methods. A de novo repetitive element database was built using four de novo software packages, including RepeatScout (v1.0.5) ${ }^{42}$, LTR_FINDER (v1.05) ${ }^{43}$, MITE-Hunter (20100819) ${ }^{44}$, and PILER-DF (v1.0) ${ }^{45}$. The de novo library was merged with Repbase $19.60^{46}$ and classified into different categories with the PASTEClassifier.py ${ }^{47}$ script embedded in REPET (v2.5) ${ }^{48}$. RepeatMasker (version open-4.0.5) ${ }^{49}$ was used to identify repetitive elements using the combined library with parameters -nolow, -no_is, -norna, -engine wublast, -qq, and -frag 20000.

\section{Gene prediction and functional annotation}

GeMoMa (v1.3.1) $)^{50}$ was used for homology prediction with the parameter evalue $=0.00001$. Five de novo gene prediction software packages were used, including Genscan (hollywood.mit.edu/GENSCAN.html), Augustus $(\mathrm{v} 2.4)^{51}$, GeneID (1.4) $)^{52}$, SNAP (v2006-07-28) ${ }^{53}$ and GlimmerHMM (v3.0.4) ${ }^{54}$. Parameters in Augustus were trained with unigenes assembled from pooled RNA-seq data. For RNA-seq based prediction, NGS transcripts and full-length transcripts were used. NGS transcripts were

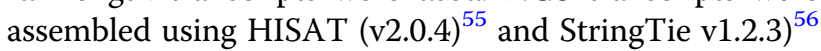
and then aligned to the genome assembly using BLAT ${ }^{57}$ with the parameters identity $\geq 0.95$ and coverage $\geq 0.90$. Unigenes were filtered using PASA (v2.0.4) ${ }^{58}$. Clean RNAseq reads were mapped to the assembled genome using TopHat ${ }^{39}$, and transcripts were assembled using Cufflinks $(\mathrm{v} 2.1 .1)^{39}$. TransDecoder $(\mathrm{v} 2.0)^{59}$ and GeneMarkS-T $(\mathrm{v} 5.1)^{60}$ were used to identify the gene structure. EVidenceModeler (EVM, v1.1.1) ${ }^{61}$ was used to obtain an integrated gene set from the three prediction strategies above with different weight settings. The final gene set was obtained after filtering out coding sequences (CDS) shorter than $300 \mathrm{bp}$ with frameshift mutations or premature stop codons. Functional annotation of the final gene set was performed using BLASTP (E-value $1 \mathrm{e}^{-5}$ ) embedded in the blast + package (v2.2.31) ${ }^{62}$ against multiple databases, including $\mathrm{KEGG}^{63}$, Swiss-Prot ${ }^{64}$, $\mathrm{TrEMBL}^{64}$, and NCBI $\mathrm{nr}^{65}$. GO annotations were assigned using the BLAST2GO pipeline $(\mathrm{v} 2.5)^{66}$. The newly annotated genes were named based on the following conventions: Bra for Brassica rapa, followed by $\mathrm{C}$ for chinensis, then the chromosome number and the letter "g" for gene. The six digits after "g" were assigned based on the gene's position relative to the top of the chromosome.

\section{Non-coding RNA (ncRNA) predictions}

tRNAscan-SE (v2.0) ${ }^{67}$ was used to predict tRNAs with two embedded searching methods (tRNA-scan and EufindtRNA). tRNAs located in repetitive regions were excluded, and tRNAs with prediction scores over 20 were retained. miRNAs were identified by a homology search against miRBase (release 22) ${ }^{68}$ with one mismatch allowed. miRDeep $2^{69}$ was used to predict secondary structures, and miRNAs with hairpin structure were retained. Other ncRNAs were predicted based on an Infernal $(\mathrm{v} 1.1 .2)^{70}$ search against the Rfam $(\mathrm{v} 12.1)^{71}$ database with default parameters.

\section{Pseudogene prediction}

GenBlastA (v1.0.4) ${ }^{72}$ with the parameter -e 1e-5 was used to identify homologous sequences in the genome, and Genewise (v2.4.1 $)^{73}$ with the parameters -both and -pseudo was used to identified pseudogenes when premature stop codons or frameshift mutations were present in homologous sequences with $60 \%$ identity and $60 \%$ coverage.

\section{Gene family clustering}

Proteins from 14 genomes were used for gene family clustering. Proteins from $A$. thaliana (TRAI10.1) were downloaded from https://www.arabidopsis.org/. Proteins from Brassica nigra, Thellungiella parvula, Thellungiella halophila, Leavenworthia alabamica, Capsella rubella, Brassica oleracea (v1.1), Brassica rapa Chiifu (v3.0), and Aethionema arabicum were downloaded from http:// brassicadb.org/brad/datasets/pub. Proteins from Cardamine hirsuta (v1.0) were downloaded from http://chi. mpipz.mpg.de/assembly.html. Proteins from Brassica rapa $\mathrm{Z} 1$ were downloaded from http://www.genoscope. cns.fr/plants. Proteins from Carica papaya (Papaya1.0) and Arabidopsis lyrata subsp. lyrata (v.1.0) were downloaded from NCBI. Only the longest transcript of each protein was used. OrthoMCL (v2.0.9; mcl inflation factor $1.5)^{74}$ was used to cluster gene families. All-against-all BLASTP searches (Blast + version 2.3.0) ${ }^{62}$ were performed with a $P$-value cutoff of $1 \mathrm{e}^{-5}$ and a minimum match length of $50 \%$.

\section{Phylogenetic tree construction and divergence time estimation}

Single-copy genes were aligned using MUSCLE (v3.8.31) 75 $^{75}$ and concatenated into one super-gene sequence for each plant genome. A maximum likelihood phylogenetic tree was constructed from the aligned protein sequences using 
PhyML $4.0^{76}$ with the parameters --sequential, --multiple 1 , --pars, --bootstrap 100, --model JTT, -f m, -t e, --pinv e, --nclasses 4, --use median, and --no_memory_check. MCMCTree implemented in the PAML package (v4.7b) ${ }^{77}$ was used to estimate speciation times.

\section{Expansion and contraction of gene families}

CAFE (v2.0) ${ }^{78}$ was used to infer gene family sizes in the most recent common ancestor (MRCA) and to determine the significance of gene family expansion/contraction based on the phylogenetic tree topology. The birth and death parameter $(\lambda)$ was 0.002 , and the $P$-value was 0.01 .

\section{Synteny and 4DTv analysis}

The BLASTP program was used to identify orthologous and paralogous genes. MCscan $\mathrm{X}^{79}$ was used to recognize syntenic blocks with parameters E_VALUE $=1 \mathrm{e}-05$, MAX GAPS $=25$, and MATCH_SIZE $=5$. Syntenic blocks were visualized with MCscan, and chromosome lengths were not scaled. The 4DTv value of each gene pair was calculated and then corrected using the HKY model ${ }^{80}$. The Ks value of each syntenic gene pair was calculated using the yn00 program in the PAML package ${ }^{77}$. Chromosome-scale syntenic block plots and dotplot were constructed using the python version of MC scan (https:// github.com/tanghaibao/jcvi/wiki/MCscan).

\section{Identification of PAV sequences and PAV clusters}

PAV sequences in the genomes of $B$. rapa NHCC001, $B$. rapa Chiifu, and $B$. rapa $\mathrm{Z} 1$ were identified using a sliding-window method as described previously ${ }^{81}$.

\section{Identification of ascorbic acid-related and glucosinolate- related genes}

A. thaliana AsA-related and GSL-related genes have been reported and were used as the set of reference genes in this study ${ }^{5,82-86}$. Their protein sequences were aligned with corresponding protein sets from the $B$. rapa genome using BLASTP (E-value $\leq 1 \times 10^{-10}$, identity $\geq 55$ ).

\section{Acknowledgements}

The authors thank Aimei Bai for assistance with the bioinformatics analysis. This work was supported by the National Key Research and Development Program (2016YFD0101701), the National Vegetable Industry Technology System (CARS23-A-06), the National Natural Science Foundation of China (31872106), and the National Key Programs for Bok Choy Breeding of China (111821301354052283). This work was also supported by the Bioinformatics Center of Nanjing Agricultural University.

\section{Author contributions}

X.L.H. conceived the project; G.F.L. and Y.L. collected the samples; G.F.L., L.M.M., F.C., and Y.L. analyzed the data; T.K.L., C.W.Z., D.X., and H.K.Z. participated in discussions and provided valuable advice; G.F.L. and Y.L. wrote and revised the manuscript.

\section{Data availability}

All of the Illumina and Nanopore sequencing data have been deposited in the Sequence Read Archive database under NCBI BioProject ID PRJNA645752. The whole-genome assembly and annotation data are publically available at: https://www.tbirs.cn/NHCCDB/Genome.jsp.

Conflict of interest

The authors declare that they have no conflict of interest.

Supplementary Information accompanies this paper at (https://doi.org/ 10.1038/s41438-020-00449-z).

Received: 24 June 2020 Revised: 28 November 2020 Accepted: 9 December 2020

Published online: 28 December 2020

References

1. Nagahara, U. Genome analysis in Brassica with special reference to the experimental formation of B. napus and peculiar mode of fertilization. Jpn. J. Bot. 7, 389-452 (1935).

2. Qi, X. et al. Genomic inferences of domestication events are corroborated by written records in Brassica rapa. Mol. Ecol. 26, 3373-3388 (2017).

3. Karam, M. A., Morsi, Y. S., Sammour, R. H. \& Ali, M. R. Assessment of genetic relationships within Brassica rapa subspecies based on polymorphism. Int. J. Curr. Microbiol. Appl. Sci. 6, 22-28 (2010)

4. Arabidopsis Genome Initiative. Analysis of the genome sequence of the flowering plant Arabidopsis thaliana. Nature 408, 796-815 (2000).

5. $\mathrm{Yu}$, J. et al. A draft sequence of the rice genome (Oryza sativa L. ssp. indica). Science 296, 79-92 (2002).

6. Chen, F. et al. The sequenced angiosperm genomes and genome databases. Front Plant Sci. 9, 418 (2018).

7. Treffer, R. \& Deckert, V. Recent advances in single-molecule sequencing. Curr. Opin. Biotechnol. 21, 4-11 (2010).

8. Jiao, Y. et al. Improved maize reference genome with single-molecule technologies. Nature 546, 524-527 (2017).

9. Schmidt, M. H. et al. De novo assembly of a new Solanum pennellii accession using nanopore sequencing. Plant Cell 29, 2336-2348 (2017).

10. Wang, $X$. et al. The genome of the mesopolyploid crop species Brassica rapa. Nat. Genet. 43, 1035-1039 (2011).

11. Zhang, L. et al. Improved Brassica rapa reference genome by single-molecule sequencing and chromosome conformation capture technologies. Hortic. Res. 5, 50 (2018).

12. Belser, $\mathrm{C}$. et al. Chromosome-scale assemblies of plant genomes using nanopore long reads and optical maps. Nat. Plants 4, 879-887 (2018).

13. Franzke, A., Lysak, M. A., Al-Shehbaz, I. A., Koch, M. A. \& Mummenhoff, K. Cabbage family affairs: the evolutionary history of Brassicaceae. Trends Plant Sci. 16, 108-116 (2011).

14. Lagercrantz, U. \& Lydiate, D. J. Comparative genome mapping in Brassica. Genetics 144, 1903-1910 (1996).

15. Tank, D. C. et al. Nested radiations and the pulse of angiosperm diversification: increased diversification rates often follow whole genome duplications. New Phytol. 207, 454-467 (2015).

16. Schranz, M. E., Lysak, M. A. \& Mitchell-Olds, T. The ABC's of comparative genomics in the Brassicaceae: building blocks of crucifer genomes. Trends Plant Sci. 11, 535-542 (2006)

17. Cheng, F. et al. Biased gene fractionation and dominant gene expression among the subgenomes of Brassica rapa. PloS ONE 7, e36442 (2012).

18. Cheng, F. et al. Deciphering the diploid ancestral genome of the Mesohexaploid Brassica rapa. Plant Cell 25, 1541-1554 (2013).

19. Cheng, F., Wu, J., Fang, L. \& Wang, X. Syntenic gene analysis between Brassica rapa and other Brassicaceae species. Front Plant Sci. 3, 198 (2012).

20. Yu, X. et al. QTL mapping of leafy heads by genome resequencing in the RIL population of Brassica rapa. PloS ONE 8, e76059 (2013).

21. Mao, Y. et al. MicroRNA319a-targeted Brassica rapa ssp. pekinensis TCP genes modulate head shape in Chinese cabbage by differential cell division arrest in leaf regions. Plant Physiol. 164, 710-720 (2014).

22. Liang, J., Liu, B., Wu, J., Cheng, F. \& Wang, X. Genetic variation and divergence of genes involved in leaf adaxial-abaxial polarity establishment in Brassica rapa. Front Plant Sci. 7, 94 (2016).

23. Pekker, I., Alvarez, J. P. \& Eshed, Y. Auxin response factors mediate Arabidopsis organ asymmetry via modulation of KANADI activity. Plant cell 17, 2899-2910 (2005). 
24. Eshed, Y., Baum, S. F., Perea, J. V. \& Bowman, J. L. Establishment of polarity in lateral organs of plants. Curr. Biol. 11, 1251-1260 (2001).

25. Eshed, Y., Izhaki, A., Baum, S. F., Floyd, S. K. \& Bowman, J. L. Asymmetric leaf development and blade expansion in Arabidopsis are mediated by KANADI and YABBY activities. Development 131, 2997-3006 (2004).

26. Ren, J. et al. Comparison of ascorbic acid biosynthesis in different tissues of three non-heading Chinese cabbage cultivars. Plant Physiol. Biochem 73, 229-236 (2013).

27. Fahey, J. W., Zalcmann, A. T. \& Talalay, P. The chemical diversity and distribution of glucosinolates and isothiocyanates among plants. Phytochemistry 56, 5-51 (2001).

28. BrianClarke, D. Glucosinolates, structures and analysis in food. Anal. Methods 2, 310-325 (2010).

29. Yang, B. \& Quiros, C. F. Survey of glucosinolate variation in leaves of Brassica rapa crops. Genet Resour. Crop Evol. 57, 1079-1089 (2010).

30. Li, J., Hansen, B. G., Ober, J. A., Kliebenstein, D. J. \& Halkier, B. A. Subclade of flavin-monooxygenases involved in aliphatic glucosinolate biosynthesis. Plant Physiol. 148, 1721-1733 (2008).

31. Rask, L. et al. Myrosinase: gene family evolution and herbivore defense in Brassicaceae. Plant Mol. Biol. 42, 93-113 (2000).

32. Cheng, F. et al. Subgenome parallel selection is associated with morphotype diversification and convergent crop domestication in Brassica rapa and Brassica oleracea. Nat. Genet. 48, 1218-1224 (2016).

33. Murray, M. G. \& Thompson, W. F. Rapid isolation of high molecular weight plant DNA. Nucleic Acids Res. 8, 4321-4325 (1980).

34. Koren, S. et al. Canu: scalable and accurate long-read assembly via adaptive kmer weighting and repeat separation. Genome Res. 27, 722-736 (2017).

35. Walker, B. J. et al. Pilon: an integrated tool for comprehensive microbial variant detection and genome assembly improvement. PLOS ONE 9, e112963 (2014).

36. Xie, T. et al. De Novo Plant genome assembly based on chromatin interactions: a case study of Arabidopsis thaliana. Mol. Plant 8, 489-492 (2015).

37. Servant, N. et al. HiC-Pro: an optimized and flexible pipeline for $\mathrm{Hi}-\mathrm{C}$ data processing. Genome Biol. 16, 259 (2015)

38. English, A. C. et al. Mind the gap: upgrading genomes with Pacific Biosciences RS long-read sequencing technology. PLOS ONE 7, e47768 (2012).

39. Trapnell, $C$. et al. Differential gene and transcript expression analysis of RNAseq experiments with TopHat and Cufflinks. Nat. Protoc. 7, 562-578 (2012).

40. Li, H. Aligning sequence reads, clone sequences and assembly contigs with BWA-MEM. Genomics 1303. Preprint at https://arxiv.org/abs/1303.3997 (2013).

41. Simão, F. A. et al. BUSCO: assessing genome assembly and annotation completeness with single-copy orthologs. Bioinformatics 31, 3210-3212 (2015).

42. Price, A. L., Jones, N. C. \& Pevzner, P. A. De novo identification of repeat families in large genomes. Bioinformatics 21, i351 (2005).

43. Xu, Z.\&Wang, H. LTR_FINDER: an efficient tool for the prediction of full-length LTR retrotransposons. Nucleic Acids Res. 35, W265-W268 (2007).

44. Han, Y. \& Wessler, S. R. MITE-Hunter: a program for discovering miniature inverted-repeat transposable elements from genomic sequences. Nucleic Acids Res. 38, e199 (2010).

45. Edgar, R. C. \& Myers, E. W. PILER: identification and classification of genomic repeats. Bioinformatics 21, i152 (2005).

46. Bao, W., Kojima, K. K. \& Kohany, O. Repbase Update, a database of repetitive elements in eukaryotic genomes. Mob. DNA 6, 11 (2015).

47. Hoede, C. et al. PASTEC: an automatic transposable element classification tool. PLOS ONE 9, e91929 (2014).

48. Timothée, F., Elodie, D., Catherine, F. \& Hadi, Q. Considering transposable element diversification in de novo annotation approaches. PLOS ONE 6, e16526 (2011)

49. Chen, N. Using RepeatMasker to identify repetitive elements in genomic sequences. Curr. Protoc. Bioinformatics. 5, 4-10 (2004).

50. Keilwagen, J. et al. Using intron position conservation for homology-based gene prediction. Nucleic Acids Res. 44, e89 (2016).

51. Nachtweide, S. \& Stanke, M. Multi-genome annotation with AUGUSTUS. Methods Mol. Biol. 1962, 139-160 (2019).

52. Blanco, E., Parra, G. \& Guigó, R. Using geneid to identify genes. Curr. Protoc. Bioinform. 64, e56 (2018).

53. Korf, I. Gene finding in novel genomes. BMC Bioinform. 5, 59 (2004).

54. Majoros, W. H., Pertea, M. \& Salzberg, S. L. TigrScan and GlimmerHMM: two open source ab initio eukaryotic gene-finders. Bioinformatics 20, 2878-2879 (2004).

55. Kim, D., Langmead, B. \& Salzberg, S. L. HISAT: a fast spliced aligner with low memory requirements. Nat. Methods 12, 357-360 (2015).
56. Pertea, M. et al. StringTie enables improved reconstruction of a transcriptome from RNA-seq reads. Nat. Biotechnol. 33, 290-295 (2015).

57. Kent, W. J. BLAT-the BLAST-like alignment tool. Genome Res. 12, 656-664 (2002).

58. Haas, B. J. et al. Improving the Arabidopsis genome annotation using maximal transcript alignment assemblies. Nucleic Acids Res. 31, 5654-5666 (2003).

59. Grabherr, M. G. et al. Full-length transcriptome assembly from RNA-Seq data without a reference genome. Nat. Biotechnol. 29, 644 (2011).

60. Tang, S., Lomsadze, A. \& Borodovsky, M. Identification of protein coding regions in RNA transcripts. Nucleic Acids Res. 43, e78 (2015).

61. Haas, B. J. et al. Automated eukaryotic gene structure annotation using EVidenceModeler and the program to assemble spliced alignments. Genome Biol. 9, R7 (2008).

62. Camacho, C. et al. BLAST+: architecture and applications. BMC Bioinform. 10, 421 (2009).

63. Kanehisa, M., Goto, S., Kawashima, S., Okuno, Y. \& Hattori, M. The KEGG resource for deciphering the genome. Nucleic Acids Res. 32, D277-D280 (2004).

64. UniProt Consortium T. UniProt: the Universal Protein knowledgebase. Nucleic Acids Res. 46, 2699 (2018).

65. Deng, $Y$. et al. Integrated nr Database in protein annotation system and its localization. Computer Eng. 32, 71-72 (2006).

66. Conesa, A. et al. Blast2GO: a universal tool for annotation, visualization and analysis in functional genomics research. Bioinformatics 21, 3674-3676 (2005).

67. Lowe, T. M. \& Chan, P. P. tRNAscan-SE On-line: integrating search and context for analysis of transfer RNA genes. Nucleic Acids Res. 44, W54-W57 (2016).

68. Kozomara, A. \& Griffithsjones, S. miRBase: annotating high confidence microRNAs using deep sequencing data. Nucleic Acids Res. 42, D68-D73 (2014).

69. Friedländer, M. R., Mackowiak, S. D., Li, N., Chen, W. \& Rajewsky, N. miRDeep2 accurately identifies known and hundreds of novel microRNA genes in seven animal clades. Nucleic Acids Res. 40, 37-52 (2012).

70. Nawrocki, E. P. \& Eddy, S. R. Infernal 1.1: 100-fold faster RNA homology searches. Bioinformatics 29, 2933-2935 (2013).

71. Gardner, P. P. et al. Rfam: updates to the RNA families database. Nucleic Acids Res. 37, D136-D140 (2009).

72. She, R., Chu, J. S., Wang, K., Pei, J. \& Chen, N. GenBlastA: enabling BLAST to identify homologous gene sequences. Genome Res. 19, 143-149 (2009).

73. Birney, E. \& Durbin, R. Using GeneWise in the Drosophila annotation experiment. Genome Res 10, 547-548 (2000).

74. Li, L., Stoeckert, C. J. \& Roos, D. S. OrthoMCL: identification of ortholog groups for eukaryotic genomes. Genome Res. 13, 2178-2189 (2003).

75. Edgar, R. C. MUSCLE: multiple sequence alignment with high accuracy and high throughput. Nucleic Acids Res. 32, 1792-1797 (2004).

76. Guindon, S. et al. New algorithms and methods to estimate maximumlikelihood phylogenies: assessing the performance of PhyML 3.0. Syst. Biol. 59, 307-321 (2010).

77. Yang, Z. PAML 4: phylogenetic analysis by maximum likelihood. Mol. Biol. Evol. 24, 1586-1591 (2007).

78. De Bie, T., Cristianini, N., Demuth, J. P. \& Hahn, M. W. CAFE: a computational tool for the study of gene family evolution. Bioinformatics 22, 1269-1271 (2006).

79. Wang, Y. et al. MCScanX: a toolkit for detection and evolutionary analysis of gene synteny and collinearity. Nucleic Acids Res. 40, e49 (2012).

80. Hasegawa, M., Kishino, H. \& Yano, T. Dating of the human-ape splitting by a molecular clock of mitochondrial DNA. J. Mol. Evol. 22, 160-174 (1985).

81. Sun, S. et al. Extensive intraspecific gene order and gene structural variations between Mo17 and other maize genomes. Nat. Genet. 50, 1289-1295 (2018).

82. Sønderby, I. E., Geu-Flores, F. \& Halkier, B. A. Biosynthesis of glucosinolatesgene discovery and beyond. Trends Plant Sci. 15, 283-290 (2010).

83. Bednarek, P. et al. A glucosinolate metabolism pathway in living plant cells mediates broad-spectrum antifungal defense. Science 323, 101-106 (2009).

84. Grubb, C. D. \& Abel, S. Glucosinolate metabolism and its control. Trends Plant Sci. 11, 89-100 (2006).

85. Duan, W. et al. Patterns of evolutionary conservation of ascorbic acid-related genes following whole-genome triplication in Brassica rapa. Genome Biol. Evol. 7, 299-313 (2014).

86. Wang, J. et al. Insights into the species-specific metabolic engineering of glucosinolates in radish (Raphanus sativus L.) based on comparative genomic analysis. Sci. Rep. 7, 16040 (2017). 